Western Washington University

Western CEDAR

2005

\title{
Getting Organized at the Nanoscale with Thermotropic Liquid Crystal Solvents
}

David L. Patrick

Western Washington University, david.patrick@wwu.edu

F. Scott Wilkinson

Western Washington University

Tiffany L. Fegurgur

Western Washington University

Follow this and additional works at: https://cedar.wwu.edu/chemistry_facpubs

Part of the Chemistry Commons

\section{Recommended Citation}

David L. Patrick, F. Scott Wilkinson, Tiffany L. Fegurgur, "Getting organized at the nanoscale with thermotropic liquid crystal solvents", Proc. SPIE 5936, Liquid Crystals IX, 59360A (22 August 2005); doi: 10.1117/12.613665; https://doi.org/10.1117/ 12.613665 


\section{Getting organized at the nanoscale with thermotropic liquid crystal solvents}

David L. Patrick, F. Scott Wilkinson, Tiffany L. Fegurgur

David L. Patrick, F. Scott Wilkinson, Tiffany L. Fegurgur, "Getting organized at the nanoscale with thermotropic liquid crystal solvents," Proc. SPIE 5936, Liquid Crystals IX, 59360A (22 August 2005); doi: 10.1117/12.613665

SPIE. Event: Optics and Photonics 2005, 2005, San Diego, California, United States 


\title{
Getting Organized at the Nanoscale with Thermotropic Liquid Crystal Solvents
}

\author{
David L. Patrick ${ }^{\dagger}$, F. Scott Wilkinson, and Tiffany L. Fegurgur. Department of Chemistry, Western \\ Washington University, 516 High St., Bellingham, WA 98225
}

\begin{abstract}
This paper summarizes recent progress toward an emerging, unconventional application of thermotropic liquid crystals (LCs) - their use as solvents for controlling the assembly of non-LC nanoscale building blocks. LCs offer a number of potential advantages compared to conventional isotropic solvents, including the ability to influence building block orientation and other structural properties. Strategies are reviewed for the exploitation of LC media to engineer order in a range of systems, including oriented organic monolayers, chiral films, and nanometer-scale particles.
\end{abstract}

\section{Introduction.}

Preparation of new materials with designer architectures at nanometer to micron length scales is the object of much current research in materials science, chemistry, and the emerging field of crystal engineering. ${ }^{1}$ A guiding principle of these efforts is the concept that by controlling order one can produce systems with tailored physical and chemical properties. This is especially true for thin films of organic compounds and small particles, where electronic, optical, and chemical properties are strongly correlated to their organization. ${ }^{2}$ Within this context, thermotropic liquid crystals (LCs), long studied for their special optical properties, have recently begun to emerge as potential candidates in a new application: as solvent hosts for controlling order among non-LC, nanometer-scale building blocks. This paper briefly reviews recent progress toward developing LC-based strategies for such applications.

Thermotropic LC solvents offer several advantages over conventional liquid media for solution phase synthesis of solid materials. These advantages stem from four characteristics unique to LC fluids: (i) They undergo directional coupling to solid surfaces, called anchoring ${ }^{3}$; (ii) They possess anisotropic (direction-dependent) properties, including various transport, optical and mechanical properties ${ }^{4}$; (iii) Orientational and other forms of order in a LC fluid can be conveniently manipulated by several methods, including external fields ${ }^{5}$; (iv) LCs exhibit a wide range of symmetry properties, many of which can, in principle, be conferred to building blocks undergoing assembly in a LC host. Each of these characteristics can be exploited in different ways to control the structure and organization of a material prepared using LC solvents.

Research in our lab and elsewhere has just begun to explore the prospects of LC solvents for engineering order among nanoscale building blocks, yet their potential for controlling organization in ways not previously possible is already becoming clear. Chief among the attractions of thermotropic LCs for this purpose are the comparative simplicity of processing techniques, and the diversity of different building blocks whose order can be controlled. We review examples ranging from control over molecular orientation in organic monolayers, to the organization of small particles such as carbon nanotubes, to control over chiral symmetry breaking, all in films prepared simply by casting from an oriented LC solvent.

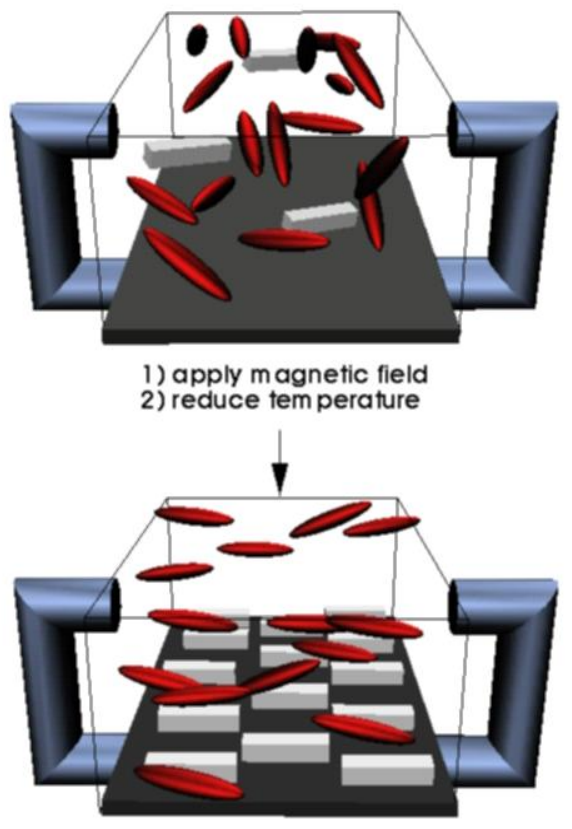

Fig. 1 In the liquid crystal imprinting technique, nm-sized building blocks are dispersed in a LC solvent, then deposited onto a supporting substrate in the presence of an alignment field. Building block orientation is controlled in part by the solvent. From Ref. 6

${ }^{\dagger}$ To whom correspondence should be addressed: Patrick@chem.wwu.edu, Tel: 360-650-3128, Fax: 360-650-2826

Liquid Crystals IX, edited by lam-Choon Khoo, Proceedings of SPIE Vol. 5936

(SPIE, Bellingham, WA, 2005) · 0277-786X/05/\$15 - doi: 10.1117/12.613665 


\section{Liquid Crystal Imprinting.}

The technique of using thermotropic LC solvents to prepare organized materials composed of non-LC building blocks was first demonstrated by Mougous et al, who called the method "liquid crystal imprinting" (LCI). ${ }^{6}$ The general approach is illustrated in Fig. 1. When a small quantity of a molecular solute (the building block) was dissolved in a LC, they found it was possible to imprint nematic (uniaxial) order on thin films grown at the LC-solid interface. When prepared under an alignment influence such as a magnetic field or rubbed polymer alignment layer, deposited films develop uniform orientational order up to macroscopic length scales.

The LCI method has several notable characteristics. One of these is that the alignment influence (for example an external magnetic field) need not exert any direct influence on the building block at all; the field aligns the LC solvent, which in turn aligns the growing film. This permits the materials engineer to begin to separate the processes of building block design from building block assembly, treating the two as isolated optimization problems. The advantage compared to methods based on self-assembly and molecular recognition is an increased freedom to focus on the properties of the building block intrinsic to its intended function (e.g. its optical, electronic or mechanical properties, etc.), rather than on the incorporation of adjunct attributes required only for the assembly process.

LCI has been shown useful in a diverse group of systems and is expected to be applicable to many more. About 75,000 thermotropic LC compounds are known, spanning a wide range of chemical properties, transition temperatures and symmetry types. This is important both because it guarantees LC solvents can be found compatible with most building block chemistries, and because it provides for a second element of control over film structure, by tuning the chemical characteristics of the solvent to influence crystal packing, defect density, inclusions and other factors. ${ }^{7}$

The main limitations of LCI stem from the forces and energies that characterize the LC state, which are usually very small. This restricts the scope of structural properties LC solvents can be used to influence. For example, the anchoring energy - a measure of the anisotropy in the surface tension at a LC interface - is typically $10^{-5} \sim 10^{-7} \mathrm{~J} \mathrm{~m}^{-2}$, up to several orders of magnitude smaller than the energy difference between different faces of most organic crystals. LC forces by themselves are therefore not expected to influence properties such as crystal growth habit. Rather, the solvent becomes important when two or more configurations of building blocks have nearly the same energies. In such cases a LC can be used to break the degeneracy, producing assemblies with controlled organization.

\section{Controlling Molecular Orientation in Organic Thin Films.}

As a first example, we discuss the use of nematic LCs to control molecular orientation in organic monolayers. Figure 2 presents a representative case. ${ }^{6}$ The scanning tunneling microscope (STM) image in part (a) shows a monolayer film of the fatty acid n-tetracosanoic acid on highly oriented pyrolytic graphite (HOPG) deposited from the nematic LC solvent ZLI-1565. A magnetic field, oriented parallel to the black arrow, was used to fix the orientation of the solvent director along a single macroscopic axis. In films deposited without a magnetic field, and in control samples prepared with a non-LC solvent or at a temperature where the LC solvent was isotropic, no long-range orientational order developed in the film. However when the bulk director was fixed using a magnetic field, fatty acid molecules in the monolayer tended to crystallize parallel to it. This is illustrated by the histogram in part (b) which collects STM observations of molecular orientation from numerous widely-spaced positions. The solid line running through the histogram is a fit using a model described below.

The example shown in Fig. 2 is a typical result, representative of observations made with several other solute LC solvent combinations. ${ }^{6,8}$ The degree orientational order in the organic monolayer is conveniently summarized using a scalar 2-dimensional order parameter $s=\left\langle 2 \cos ^{2}(\theta-\bar{\theta})-1\right\rangle$, where $\theta$ is the orientation of a crystallite measured by

STM, $\bar{\theta}$ is the average orientation of all crystallites, and the brackets denote an average over multiple observations. Here $s=1$ corresponds to perfect order and $s=0$ to perfect disorder. The degree of order varies between systems and depends on the deposition conditions. In the example of Fig. $2, s \sim 0.6$, but in some systems films can be prepared routinely with $s>0.9$.

Two different approaches have been developed for depositing monolayer organic films: (i) a direct deposition approach, wherein a solution of the LC / solute is applied directly to the substrate and the solute self-assembles or crystallizes at the interface. This was the method used to prepare the film in Fig. 2. (ii) A template approach wherein a sacrificial template film is deposited in a first step and displaced by the target solute in a second step. ${ }^{9}$ In the template approach, a sacrificial template monolayer engineered to possess specific orientational properties (or chiral properties see below) is prepared using the direct deposition method. Afterwards this template can be displaced by a target solute through a process of competitive adsorption, during which some symmetry characteristics of the template can be conferred to the building block. 

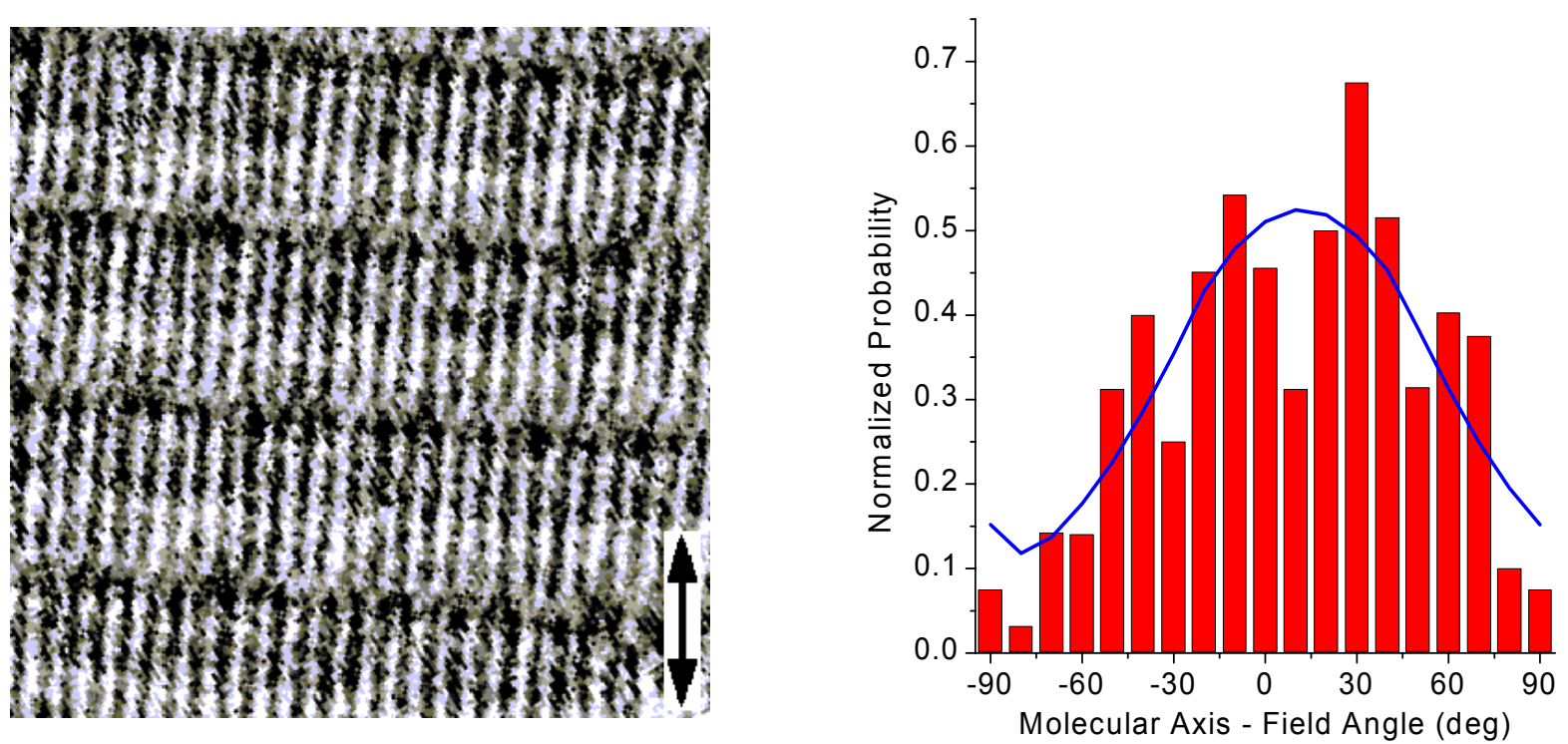

Fig. 2. (a) STM image showing a monolayer of n-tetracosanoic acid on HOPG deposited from the nematic LC ZLI-1565. Each stick-like feature is a single molecule. The arrow gives the orientation of the magnetic field and LC director. (b) Histogram showing alignment molecules based on 217 macroscopically-spaced observations on several samples. The solid line is a fit to the distribution using the model described in the text. From Ref. 6.

For the case of monolayer organic films, the mechanism for imprinting orientational order appears to be as follows: at most interfaces LCs tend to adopt a preferred orientation - the anchoring direction. ${ }^{10}$ If the anchoring direction above a nucleus differs from the director orientation in the bulk imposed by an external influence such as an electric field, strain energy is stored in the fluid as a distortion in the director, and a quasi-elastic torque is exerted on the nucleus. For planar anchoring, a misaligned nucleus leads to a twist distortion, as shown in Fig. 3. The process can be modeled by considering the alignment energy of these nuclei, which in monolayer films is approximately $U=A W / 2 \sin ^{2} \phi$, where $A$ depends on the LC twist elastic constant $K_{22}$ and nucleus size, $W$ is the anchoring energy per unit area of nucleus, and $\phi$ is the angular difference between the anchoring direction above a nucleus and the orientation of the nematic director. ${ }^{6}$ Typical values for a film of small organic molecules are: $A \sim 10^{2}-$ $10^{3} \mathrm{~nm}^{2}$, and $W \sim 10^{-23}-10^{-25} \mathrm{~J} \mathrm{~nm}^{-2}$, so the maximum cost of nucleus misalignment is approximately $10-10^{-3} \mathrm{kT}$ per nucleus at room temperature. This can be sufficient to impart a high degree of orientational order.

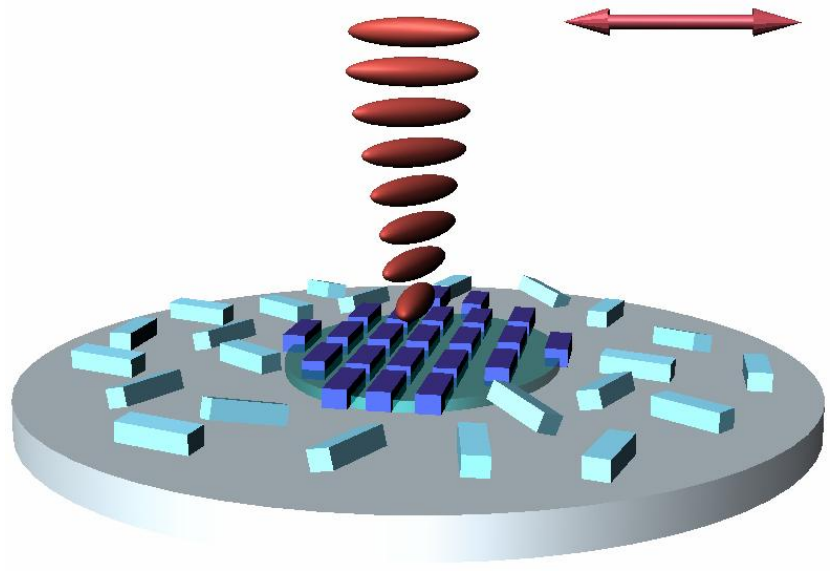

Fig. 3. If the anchoring direction above a nucleus differs from the bulk director established by the field (indicated by an arrow), a twist distortion occurs at the interface, increasing the system's orientational energy. By rotating through an angle $\phi$ the nucleus minimizes this energy. Blocks represent molecules crystallizing on a substrate; ellipsoids represent the interfacial LC fluid. 


\section{Controlling Order in Films of Nanoscale Particles}

Over the past several decades numerous nanometer to micron-sized materials have been synthesized and characterized, among them various nanoclusters, nanowires, fullerenes, quantum dots, and supramolecular assemblies. The emerging "bottom-up" approach envisions the use of these fundamental building blocks to create novel nanometer to micron-scale structures in order to harness their special physical and chemical properties.

Thermotropic LCs have been known to be capable of orienting small particles for 35 years. ${ }^{11}$ In the last 10 years, an intense effort has been focused in particular on understanding the behavior of spherical particles in nematic solvents. ${ }^{12}$ However it is only recently that much attention has been given to the concept of using LCs to organize nanoparticles for the creation of new materials. $13,14,15,16$

One attraction afforded by LCs for this application is that films of oriented particles can be prepared relatively easily, and orientation can be controlled to a very high degree. A particularly simple method for doing so is illustrated in Fig. 4. A suspension of particles in a LC is cast upon a porous membrane, which has grooves to align the director along a single axis. Draining this suspension through the membrane leaves an ordered particle film on its surface. This technique has been used for example to produce some of the most highly oriented single-wall carbon nanotubes films ever produced.

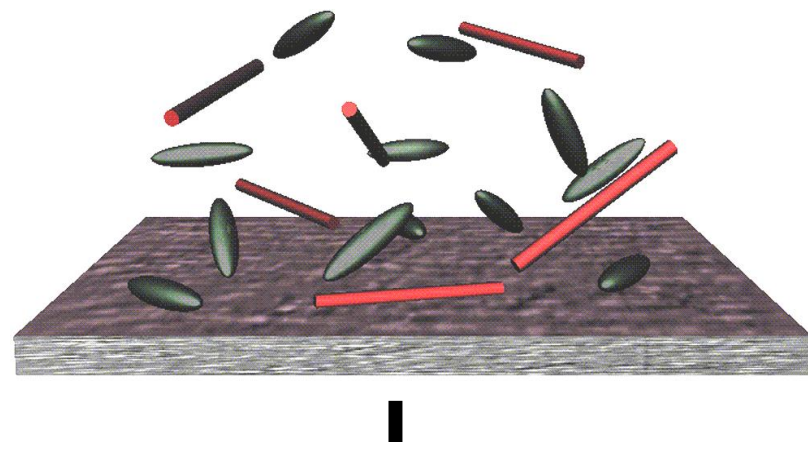

Orient with magnetic or electric field, or substrate grooves
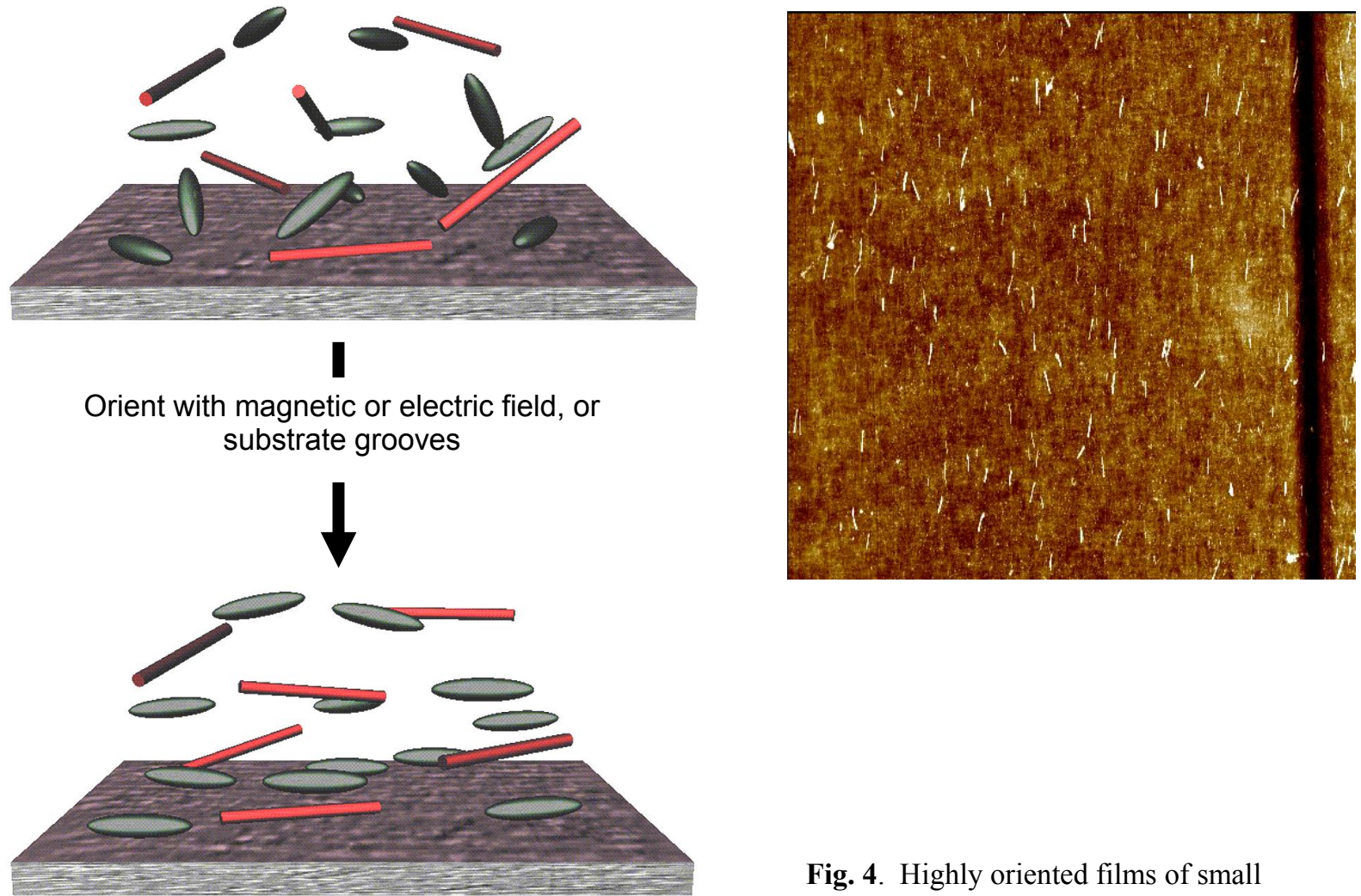

Fig. 4. Highly oriented films of small particles can be produced relatively simply using LC solvents by casting a suspension through a porous membrane filter, leaving a layer of particles on its surface. The AFM image shows a film of multi-walled carbon nanotubes produced by this method $(50 \times 50$ $\mu \mathrm{m})$. From Ref. 14.

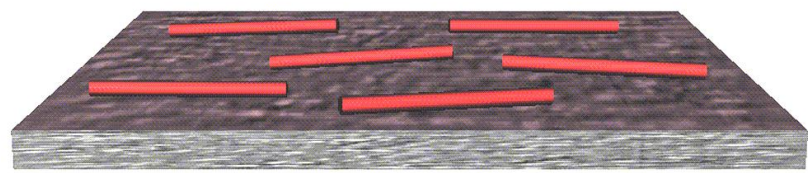


The orientation of particles in the LC solvent depends on a number of factors, including their surface chemistry and choice of LC solvent. ${ }^{13}$ By varying these parameters, more complex, multi-layered films can be prepared with, for example, alternating particle orientation between layers. Spatially inhomogeneous arrangements of particles can also be produced, using patterned electrodes. This has been demonstrated for both single- and multiwalled carbon nanotubes, where a localized electric field was used to orient the LC director and connect a pair of electrodes with carbon nanotube wiring. ${ }^{14}$

\section{Controlling Chirality in Molecular Films}

For over 150 years understanding and controlling chirality has been an important objective in natural science. ${ }^{17}$ Chirality affects the performance of many chemical, biochemical, and physical systems, and therefore chiral control has widespread application. ${ }^{18,19}$ Many compounds which are achiral in 3D can still form chiral surfaces when adsorbed to a solid substrate. In such cases chirality emerges due to a reduction in symmetry caused by either hindered conformational mobility or by specific interactions driving the formation of chiral molecular arrangements. Chirality can occur at the molecular level, or at the supramolecular level, in the form of chiral conglomerates. These phenomenon are now well established, and have been experimentally observed in dozens of different systems. ${ }^{19,20,21,22}$

On the basis of symmetry arguments, one can show that any molecule in the $\mathrm{C}_{\mathrm{nh}}, \mathrm{C}_{\mathrm{s}}$ or $\mathrm{C}_{i}$ point groups (which are achiral in 3D) has the potential to form a chiral surface if adsorbed with the proper orientation in $2 \mathrm{D} .{ }^{20,21}$ In addition, compounds with other symmetries can form chiral supramolecular patterns, even if the individual adsorbates are not themselves chiral. ${ }^{22}$ These processes however always lead to the formation of pairs of energetically equivalent enantiomorphs. Consequently, surfaces covered by multiple microscopic molecular domains are racemic on a macroscopic scale, since left- and right-handed regions form in equal numbers.

To produce films with a net excess of one enantiomorph over the other - a prerequisite for many applications of chiral surfaces - a further reduction in symmetry is required. Heretofore, such homochiral surfaces have always been produced by introducing a chiral component of some kind, such as a chiral adsorbate, reactant or catalyst, or by slicing crystals along high index faces. ${ }^{19}$ In this subsection we review how LCI can be used for the preparation of macroscopically homochiral films without any chiral inputs; instead the chiral symmetry is systematically broken using the LC solvent to induce a preferred in-plane orientation of adsorbed molecules on an achiral single crystal substrate.

Due to their uniaxial symmetry, nematic solvents are only able to directly influence orientational order in thin films. To extend control to include additional elements of order, additional constraining influences are required. The additional influence is provided by the substrate, which through careful experimental design combines with the LC solvent to create conditions in which a single enantiomer minimizes the energy.

To understand how this occurs, consider the object and its mirror image in Fig. 5, shown adsorbed on the (0001) graphite surface. The object belongs in the $\mathrm{C}_{2 \mathrm{~h}}$ point group and hence in $3 \mathrm{D}$ is achiral, but in 2D it is chiral, because it cannot be superimposed on its mirror image without leaving the plane. ${ }^{23}$ Suppose that this object forms a crystalline monolayer, with the stick-like tail in registry with the substrate. On graphite (0001) with its 3-fold symmetry, there are six energetically equivalent ways to arrange the object (3 substrates orientations) $\mathrm{x}(2$ enantiomorphs). In the absence of any other influence, all six configurations would be degenerate, and a macroscopic film would be racemic. If a uniaxial alignment influence such as that created by a nematic LC solvent is applied which causes one in-plane orientation to be more favored than others, there will generally exist just one configuration - and hence one chirality - which orients the object most nearly parallel to the influence, while maintaining registry of the tail with the substrate. Application of the uniaxial influence breaks in-plane orientational symmetry, and hence chiral symmetry as well, producing a macroscopically chiral film. Moreover, by varying the in-plane orientation of the uniaxial influence, configurations of either absolute chirality can be selected by design, as can the enantiomeric

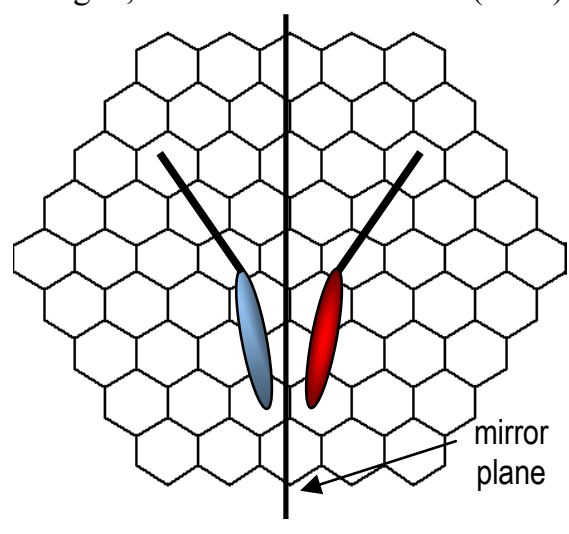

Fig. 5. An object which is achiral in $3 D$, but which can form a chiral surface in $2 D$. The vertical line represents a mirror plane. excess, by orienting the uniaxial influence part way between two minima.

The first demonstration of chiral control via LCI involved a film of the compound 4'-octyl-4-cyanobiphenyl (8CB) on single crystal graphite. When placed in contact with graphite $8 \mathrm{CB}$ forms a single, polycrystalline monolayer at the interface between the substrate and bulk fluid supernate. ${ }^{24}$ Molecules in the monolayer organize as shown in Fig 6, with the long molecular axis lying in the substrate plane. The unit cell contains eight molecules, in registry with the 
substrate, and aligned in an antiparallel configuration resulting in a cancellation of dipoles. Registry is driven by an interaction between the alkyl tail and graphite; tails always orient along one of the three graphite $<1100>$ directions of a form. ${ }^{25}$ In 3-dimensions $8 \mathrm{CB}$ is achiral, but when the molecule is confined to two dimensions upon adsorption, hindered conformational mobility, combined with the presence of the substrate, breaks the mirror symmetry and surfaces become chiral. The two enantiomorphs phase separate into purely left- or right-handed domains measuring a few hundred nanometers in size.

$8 \mathrm{CB}$ is also a room temperature smectic-A liquid crystal. The system therefore represented the simplest implementation of the concept proposed above, in which the adsorbate had the same identity as the LC solvent.

When an 8CB monolayer crystallizes on graphite, six energetically equivalent configurations are observed with scanning tunneling microscopy (STM): (three distinguishable orientations) x (two enantiomorphs). Figure 6 shows two of the six possible configurations; the other four are derived from these by rotating $\pm 60^{\circ}$ in the plane. All six configurations normally to occur with equal frequency, and hence in the absence of an external perturbation $8 \mathrm{CB}$ films are racemic. $^{25}$

To break the degeneracy among configurations, samples were prepared by applying a $1.2 \mathrm{~T}$ magnetic field parallel to the substrate as a droplet of $8 \mathrm{CB}$ was placed on the graphite (0001) surface, heated to above its isotropic transition temperature, then gradually cooled to room temperature. Orientational order is imprinted on the monolayer by the mechanism described in the introduction. Although strong enough to produce well-aligned films, the orientational energy of the magnetic field was small compared to the strength of intermolecular and molecule-substrate interactions, so the arrangement of $8 \mathrm{CB}$ molecules within the unit cell, and registry of molecules with the surface were unaltered from reference samples prepared in the absence of any field. The outcome of applying a magnetic field was therefore to select some of the six possible configurations as being most favored. The preferred configuration for a particular field direction was generally observed to be one which oriented the cyanobiphenyl headgroups as nearly parallel to the field as possible, while maintaining registry of the alkyl tails along one of the graphite $<1100>$ directions of a form.

When films were deposited in a magnet, one configuration could be made to be more favored - and the others less favored - by changing the field orientation. Two representative examples are shown in Fig. 6, where the field was rotated by $-19^{\circ}$ and $+19^{\circ}$ from $<1100>$, respectively. The symmetry between configurations was broken, producing a film with a net excess of one enantiomorph over the other.

The outcome of this effect is summarized in the histogram, which shows the overall enantiomeric excess $e e=\left(P_{R}-P_{L}\right) /\left(P_{R}+P_{L}\right)$ for several field orientations measured by STM. Here $P_{\mathrm{x}}$ is the experimental probability of observing a right- or left-handed domain. The enantiomeric excess oscillates between right- and left-handed surfaces with a repeat period of $60^{\circ}$. At the extrema $e e>0.6$, meaning more than $80 \%$ of the surface was covered by a single enantiomorph. Simply by changing the orientation of the field the absolute chirality and enantiomeric purity of the film could be engineered over a wide range of values.

The model outlined above in the Liquid Crystal Imprinting section can be used to quantitatively describe the variation in chirality. In an earlier study $A W / 2$ was measured and found to equal $25 \mathrm{~kJ} \mathrm{~mol}^{-1}$ in this system. ${ }^{6}$ From this the probability of observing a given configuration can be estimated by assuming an equilibrium distribution as $P_{i}=Q^{-1} \exp \left(-U_{i} / k T\right)$, where $Q=\sum_{i=1}^{6} \exp \left(-U_{i} / k T\right)$ is the

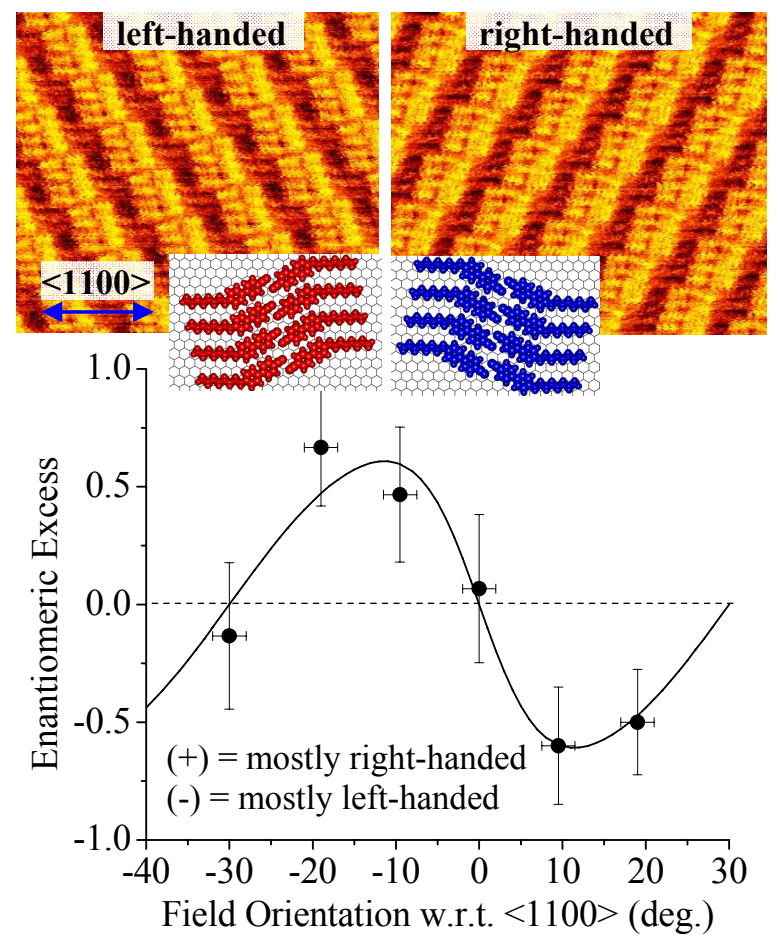

Fig. 6. Absolute chirality and enantiomeric excess in polycrystalline $8 C B$ monolayers on graphite were controlled by preparing samples in a magnetic field oriented along different directions. The top two STM images show left- and right-handed films $(15 \times 15 \mathrm{~nm})$. The plot shows average enantiomeric excess in several films prepared with different orientations of the magnetic field relative to the graphite $<1100>$ crystallographic direction. From Ref. 8. 
absolute temperature. The enantiomeric excess can therefore be computed directly from $P$, with the predicted (not fitted) result shown by the solid line in Fig. 6 .

\section{Summary.}

Recent research investigating the use of thermotropic LC solvents for preparing organized assemblies of nanoscale, non-LC building blocks demonstrate they offer a number of potential advantages compared to conventional isotropic solvents for influencing building block orientation and other structural properties. They enable comparatively simple processing techniques, can be used with a wide range of different building blocks, and are compatible with strategies for localized patterning and control over organization.

\section{Acknowledgements.}

This work was supported by the National Science Foundation (PECASE-9985428), The Camille and Henry Dreyfus Foundation, and the American Chemical Society Petroleum Research Fund (PRF-37549-B7).

1) See for instance, (a) "Crystal engineering - the design of new solids", G. R. Desiraju, Elsevier, New York, BY (1989); (b) Hierarchical Structures in Biology as a Guide for New Materials Technology", a report from the committee on synthetic hierarchical structures, National Research Council, D. A. Tirrell, Chair, National Academy Press (1994).

2) E. A. Silinsh, V. Čápek, "Organic Molecular Crystals Interaction, Localization, and Transport Phenomena", AIP Press, New York (1994).

3) (a) B. Jerome, Rep. Prog. Phys. 1991, 54, 391; (b) The Surface Physics of Liquid Crystals, A. A. Sonin, Gordon and Breach, Luxembourg (1995).

4) (a) Liquid Crystals, $2^{\text {nd }}$ Ed., Chandrasekhar, S., Cambridge University Press, Cambridge, (1992); (b) The Physics of Liquid Crystals, $2^{\text {nd }}$ Ed., De Gennes, P. G.; Prost, J., Oxford University Press, Oxford (1995).

5) (a) Dekker, A. J., Can. J. Phys. 1987, 65, 1185; (b) Dunmur, D. A.; Palffy-Muhoray, P., J. Phys. Chem. 1988, $92,1406$.

6) (a) Mougous, J.; Baker, R.; Patrick, D. L., Phys. Rev. Lett. 2000, 84, 2742; (b) Patrick, D. L., U.S. Patent Nos. 6,723,396 and $6,858,270$.

7) Davey, R. J., J. Cryst. Growth 1986, 76, 637.

8) Berg, A.; Patrick, D. L., Angew. Chemie Int. Ed. 2005, 43, 1744.

9) Hickman, S.; Hamilton, A.; Patrick, D. L., Surf. Sci., 2003, 537, 113.

10) LCs undergo three types of surface anchoring: 1) planar anchoring, in which the LC director lies in the plane of the surface; 2) homeotropic anchoring, in which the director is normal to the surface plane; 3 ) tilted anchoring, in which the director orients at an intermediate azimuthal angle.

11) Brochard, F.; de Gennes, P. G. J. de. Physique 1970, 31, 691.

12) See for example, (a) Fukuda, J.; Yokoyama, H., Phys. Rev. E 2002, 65, 041709; (b) Meeker, S. P.;. Poon, W. C. K; Crain, J. Terentjev, E. M., Phys. Rev. E, 2000, 61, R6083; (c) Lev, B. I.; Tomchuk, P. M., Phys. Rev. E 1999, 59, 591; (d) Shiyanovskii, S. V.; Kuksenok, O. V., Mol. Cryst. Liq. Cryst. 1998, 321, 45; (e) Poulin, P.; Weitz, D. A., Phys. Rev. E 1998, 57, 626; (f) Lubensky, T. C.; Pettey, D.; Currier, N.; Stark, H., Phys. Rev. E 1998, 57, 610.

13) Lynch, M. D.; Patrick, D. L. Chem. Mater. 2004, 16, 762.

14) Lynch, M. D.; Patrick, D. L. Nano Lett. 2002, 2, 1197.

15) Dierking, I.; Scalia, G.; Morales, P.; J. Appl. Phys. 2005, 97, 044309.

16) R. W. Ruhwandl, E. M. Terentjev, Phys. Rev. E 1997, 55, 2958.

17) Pasteur, L. Ann. Chim. Phys., 1848, 24, 442.

18) "Chirality in Natural and Applied Science", Lough, W. J., Wainer, I. W. (Eds.), CRC Press, Boca Raton, FL, 2002.

19) (a) Lopinski, G. P.; Moffatt, D. J.; Wayner, D.D.M.; Wolkow, R.A., Nature, 1998, 392, 909; (b) Eckhardt, C. J.; Peachy, N. M.; Swanson, D. R.; Takacs, J. M.; Khan, M. A.; Gong, X.; Kim, J.-H.; Wang, J.; Uphaus, R. A., Nature 1993, 362, 614. (c) Kühnle, A.; Linderoth, T. R.; Hammer, B.; Besenbacher, F., Nature 2002, 415, 891. (d) Switzer J.A.; Kothari, H.M.; Poizot P.; Nakanishi, S.; Bohannan, E.W. Nature 2003, 425, 463; (e) Giancarlo, L.C.; Flynn, G., Acc. Chem. Res., 2000, $33,491$.

20) (a) Qiu, X.; Ruiz-Garcia, J.; Stine, K. J.; Knobler, C. M.; Selinger, J. V., Phys. Rev. Lett. 1991, 67, 703; (b) Viswanathan, R.; Zasadzinski, J. A.; Schwartz, D. K., Nature 1994, 368, 440; (c) Kim, K.; Plass, E.; Matzger, A. J., Langmuir 2003, 19, 7149; (d) Plass, K. E.; Kim, K.; Matzger, A. J., J. Am. Chem. Soc. 2004, 126, 9042; (e) Stawasz, M. E.; Parkinson, B. A., Langmuir 2003, $19,10139$.

21) Kohl, P. B.; Patrick, D. L., J. Phys. Chem. B. 2001, 105, 8203.

22) (a) Lim, R.; Li, J.; Li, S. F. Y., Langmuir 2000, 16, 7023; (b) Charra, F.; Cousty, J., Phys. Rev. Lett. 1998, 80, 1682. (c) Li, C.; Zeng, Q.; Wu, P.; Xu, S.; Wang, C.; Qiao, Y.; Wan, L.; Bai, C., J. Phys. Chem. B 2002, 106, 13262. 
23) Note that the objects by themselves are not chiral, insofar as a free-standing film would still possess mirror symmetry. It is the combined surface (monolayer + substrate) that is chiral.

24) Foster, J. S.; Frommer, J. E., Nature 1988, 333, 542.

25) Smith, D. P. E., J. Vac. Sci. Technol. B, 1991, 9, 1119. 